\title{
Cerebrospinal fluid shunt-associated infections in pediatrics: Analysis of the epidemiology and mortality risk factors
}

\author{
Soledad González, M.D. ${ }^{a}$, Mirta Carbonaro, B.S. ${ }^{a}$, Ana G. Fedullo, M.D. ${ }^{a}$, \\ María I. Sormani, M.D. ${ }^{a}$, María del C. Ceinos, Biochemist ${ }^{b}$, Roberto González, M.D. ${ }^{c}$ \\ and María T. Rosanova, M.D. ${ }^{a}$
}

\begin{abstract}
Introduction. Infections are the most common complications of ventricular cerebrospinal fluid shunts. The objective of this study was to describe the clinical, microbiological, and evolutionary characteristics of children with ventricular cerebrospinal fluid shunt-associated infections and analyze the risk factors for mortality.

Population and methods. Descriptive, retrospective study carried out at Hospital "Prof. Dr. Juan P. Garrahan" in the Autonomous City of Buenos Aires. All patients hospitalized between January 1st, 2012 and December 31st, 2015 who were compatible with ventriculitis and had a positive cerebrospinal fluid culture were assessed.

Results. A total of 49 patients with 57 infections were included. Their median age was 62 months (interquartilerange:19-114).Malespredominated: $34(70 \%)$. A central nervous system tumor was the most common underlying disease: $20(40 \%)$. Coagulase-negative Staphylococcus was isolated in $26(46 \%)$; Staphylococcus aureus, in $13(23 \%)$; Gram-negative bacilli, in $11(19 \%)$; and other microorganism, in $7(12 \%)$. Treatment consisted of removal of ventricular shunt plus antibiotic therapy for $55(97 \%)$ infections. The mortality rate was $9 \%$. The only statistically significant factors associated with mortality were positive blood cultures $(\mathrm{p}=0.04)$, fever at the time of admission $(\mathrm{p}=0.04)$, and septic shock $(\mathrm{p}=0.0006)$.

Conclusions. Coagulase-negative Staphylococcus was the most common microorganism. Valve removal plus antibiotic therapy was the most frequently instituted treatment. Fever at the time of admission, positive blood cultures, and septic shock were predictors of mortality.

Key words: cerebral ventriculitis, mortality, child, cerebrospinal fluid shunts.
\end{abstract}

http: / / dx.doi.org/10.5546/ aap.2018.eng.198

To cite: González S, Carbonaro M, Fedullo AG, et al. Cerebrospinal fluid shunt-associated infections in pediatrics: Analysis of the epidemiology and mortality risk factors. Arch Argent Pediatr 2018;116(3):198-203.

\section{INTRODUCTION}

Ventricular cerebrospinal fluid (CSF) shunts are commonly used in pediatric neurosurgery for the treatment of hydrocephalus of various etiologies, including anatomical alterations (myelomeningocele, Arnold-Chiari malformation), prematurity with intraventricular hemorrhage, central nervous system (CNS) infections, tumors or severe traumatic brain injury. ${ }^{1}$

Infections are the main causes of morbidity and mortality ${ }^{2}$ in children undergoing ventricular CSF shunt implantation.

Reports on incidence are variable: from $0.3 \%$ to $26 \% .^{3}$ This complication is associated with the requirement of multiple surgical procedures, extended length of hospital stay, and increased hospital costs. Four mechanisms have been proposed that may promote infections: the first and most frequent mechanism is shunt colonization during the surgical procedure; the second one is retrograde infection from the distal end of the shunt, e.g., in the case of intestinal perforation; the third one is through the skin after tapping the reservoir or following an injury in the skin covering the catheter; and the fourth mechanism is hematogenous spread, in general, in patients with ventriculoatrial shunts and bacteremia. ${ }^{4}$

The factors associated with a higher risk for infection of ventricular CSF shunts include prematurity, younger age, prior infection, hydrocephalus etiology (purulent meningitis, myelomeningocele, and hemorrhage), surgeon's lack of experience, length of surgical 
procedure, inadequate skin preparation, and frequent shunt revisions, ${ }^{4}$ among others.

The causative agents of ventricular CSF shunts-associated infection are, in general, poorly virulent, cause little inflammatory reaction, and adhere to the device surface forming biofilms, thus hindering diagnosis and treatment. Symptoms are usually non-specific; most of the times, the only symptoms observed are those that may be attributed to intracranial hypertension resulting from valve dysfunction (headache, nausea, vomiting, and altered mental status). ${ }^{5}$

Mortality has been reported to be variable but, in general, low, although recent publications have described a mortality rate higher than $15 \% .{ }^{2,6} \mathrm{Few}$ pediatric studies have been conducted that assess the factors associated with mortality in this type of infections.

\section{OBJECTIVE}

To describe the epidemiological, clinical, microbiological, and evolutionary characteristics of children with ventricular CSF shunt-associated infections and analyze risk factors for mortality.

\section{MATERIALS AND METHODS}

This retrospective, descriptive, and observational study was carried out at Hospital de Pediatría "Prof. Dr. Juan P. Garrahan" in the Autonomous City of Buenos Aires between January $1^{\text {st }}, 2012$ and December 31 ${ }^{\text {st }}, 2015$. The study was approved by the Ethics Committee of the hospital.

Inclusion criteria: Children aged 1 month to 18 years with clinical manifestations compatible with ventriculitis and a positive CSF culture. The following clinical manifestations were considered compatible with ventriculitis: headache, vomiting, altered mental status, irritability, fever without another source, erythema along the valve tract, and peritoneal signs without another cause in patients with ventriculoperitoneal shunts.

Data collection: In the setting of the epidemiological surveillance conducted by a group of nurses specialized in infection management, all patients undergoing neurosurgical procedures are recorded and the rates of post-operative infections are reviewed annually to improve health care quality. Based on this database, patients who had a ventricular CSF shunt-associated infection were identified. Demographic data, underlying disease, hydrocephalus etiology, age at the moment of first shunt implantation, date of implantation and characteristics of current shunt, clinical and microbiological characteristics of CSF and blood cultures, clinical course, and medical and surgical treatment were subsequently collected by reviewing medical records.

CSF samples were processed as per the Clinical Microbiology Laboratory procedures manual and in accordance with the international recommendations and standards (ISO 15189:2007). ${ }^{7}$ A direct microscopic examination (Gram staining) was done, followed by a culture after centrifugation. Solid culture media were used (blood agar, chocolate agar). The commercial brands used to prepare culture media were Oxoid Columbia Blood Agar Base for blood and chocolate agar plates, and Biokar (France) for the liquid medium (brain-heart-infusion [BHI] broth). Solid media were incubated for 48 hours at an adequate temperature and atmosphere, whereas liquid media were incubated for 7 days at $35-37^{\circ} \mathrm{C}$. Positive cultures were processed using the VITEK ${ }^{\circledR} 2 \mathrm{C}$ automated system (bioMérieux, France) and the genus and species of isolated microorganisms, as well as antibiotic susceptibility by minimum inhibitory concentration (MIC), were determined. ${ }^{8}$

Definitions: Ventricular CSF shunt-associated infection was defined as the identification of a bacterial or fungal agent isolated in the CSF collected by shunt reservoir tapping or during the surgery, in addition to clinical signs compatible with ventriculitis.

Mortality: Mortality was assessed if occurring within 30 days of infection diagnosis.

Statistical analysis: Data were processed using the Epi-Info 6.0 software. Continuous outcome measures were reported as median and interquartile range (IQR). Categorical outcome measures were expressed in number and percentage. A $p$ value $\leq 0.05$ was considered the cut-off point for statistically significant differences.

Risk factors for mortality were assessed using a univariate analysis of studied outcome measures.

\section{RESULTS}

During the study period, 261 ventricular CSF shunts were implanted per year in average (range: 252-278). Forty-nine patients met the inclusion criteria; 8 had 2 infections in the study period, so a total of 57 infections were recorded. Their median age was 62 months (IQR: 19-114). Males predominated: 34 patients $(70 \%)$. The 
causes of hydrocephalus are described in Table 1. The median time from shunt implantation to infection was 25 days (IQR: 7-37), which was sub-divided into 4 periods: $0-30$ days, 33 patients $(57 \%)$ ); 31-60 days, 13 patients (22\%); 61-90 days, 2 patients (4\%), and $>91$ days, 6 patients $(10 \%)$.

Patients' median age at the time of implantation of the first shunt was 12 months (IQR: 1-96); 32 patients (56\%) required valve replacement, and $17(30 \%)$ had a history of pyoventriculitis.

In relation to infections, $52(91 \%)$ were observed in ventriculoperitoneal shunts; $2(4 \%)$, in ventriculoatrial shunts, and $3(4 \%)$, in other types of shunts. The most common presentations were valve dysfunction in $44(77 \%)$, fever in $27(47 \%)$, and cellulitis along the tract in $6(11 \%)$. The mean duration of fever was 2 days (range: 1-14). Pathological findings were observed in the CSF collected at the time of admission in $42(78 \%)$, with a median glucose, cellularity, and proteins of $48 \mathrm{mg} / \mathrm{mL}$ (IQRC: 31-62), 28.5 cells $/ \mathrm{mm}^{3}$ (IQR: 4-180), and $74 \mathrm{mg} / \mathrm{dL}$ (IQR: 21-179), respectively. Gram staining was positive in $6(10 \%)$ patients. Microbiological isolation in CSF corresponded to coagulase-negative Staphylococcus in $26(46 \%)$ patients, methicillin-resistant Staphylococcus aureus in $8(14 \%)$, methicillin-susceptible Staphylococcus aureus in 5 (9\%), Pseudomonas aeruginosa in $3(5 \%)$, Enterobacter spp. in $2(3 \%)$, Pseudomonas stutzeri in $2(3 \%)$, Acinetobacter baumannii in $1(2 \%)$, Stenotrophomonas maltophilia in $1(2 \%)$, Klebsiella pneumoniae in $1(2 \%)$, Achromobacter xylosoxidans in $1(2 \%)$, Candida albicans in $1(2 \%)$, and others in $6(10 \%)$. A polymicrobial culture was recorded for 1 infection $(2 \%)$. Bacteremia was observed concomitantly with the same microorganism isolated in the CSF en 6 (10\%) cases. The following microorganisms were isolated in blood cultures: coagulase-negative Staphylococcus in $2(3 \%)$, methicillin-resistant Staphylococcus aureus in 2 (3\%), methicillin-susceptible Staphylococcus aureus

TABLE 1. Cause of hydrocephalus ( $n=49$ patients)

\begin{tabular}{lc}
\hline & $\mathbf{N}(\boldsymbol{\%})$ \\
\hline CNS tumor & $20(40)$ \\
Congenital hydrocephalus & $10(20)$ \\
Anatomical malformations & $6(12)$ \\
Myelomeningocele & $5(10)$ \\
Meningitis & $4(8)$ \\
Intraventricular hemorrhage & $2(4)$ \\
Severe traumatic brain injury & $1(2)$ \\
Stroke & $1(2)$ \\
\hline
\end{tabular}

CNS: central nervous system. in $1(2 \%)$, and Achromobacter xylosoxidans in $1(2 \%)$.

The median length of hospital stay was 35 days (IQR: 23-61). Treatment consisted of removal of ventricular shunt plus antibiotic therapy for $55(97 \%)$ infections. An external ventricular shunt was required in $54(94 \%)$, with a median of 1 replacement (range: 1-3) (Table 2). It is worth noting that, in our hospital, as part of the external ventricular drain (EVD) management protocol, shunts are replaced every 7 days; however, some patients may require a replacement more often due to mechanical complications or infections.

The most commonly used antibiotic was vancomycin in $28(49 \%)$ patients. The mean treatment duration was 21 days (range: 14-26). Two (3\%) patients received only medical treatment because they were diagnosed with tumor progression and were receiving palliative care.

Following treatment, a ventricular shunt was implanted in $46(80 \%)$ patients. The median days until ventricular shunt reimplantation was 23 days (IQR: 18-30). In relation to complications, $3(5 \%)$ patients had superinfection with Gramnegative bacilli and $5(9 \%)$ died as a result of the infection. Two patients died within 48 hours after hospitalization due to septic shock; both had Staphylococcus aureus isolated in the blood culture. The 3 remaining patients died in the month following ventriculitis diagnosis and had an uncontrolled infection at the time of death. Their underlying disease was CNS tumor and had been diagnosed with concomitant relapse.

The univariate analysis showed that the only statistically significant factors associated with mortality were positive blood cultures $(p=0.04)$, fever at the time of admission $(\mathrm{p}=0.04)$, and septic shock $(p=0.0006)$.

TABLE 2. Course and treatment

\begin{tabular}{lc}
\hline & $\mathbf{N}(\%)$ \\
\hline $\begin{array}{l}\text { Length of hospitalization in days } \\
\text { Ventricular shunt removal plus } \\
\text { antibiotic therapy }\end{array}$ & 35 (IQR: 23-61) \\
$\begin{array}{l}\text { External ventricular shunt } \\
\text { Ventricular shunt reimplantation }\end{array}$ & $55(97)$ \\
$\begin{array}{l}\text { Median number of replacements } \\
\text { of external ventricular shunt }\end{array}$ & $46(84)$ \\
$\begin{array}{l}\text { Median length of antibiotic } \\
\text { therapy in days }\end{array}$ & 1 (IQR: 1-3) \\
$\begin{array}{l}\text { Median days until ventricular } \\
\text { shunt reimplantation }\end{array}$ & 21 (IQR: 14-26) \\
Mortality & 23 (IQR: 18-30) \\
\hline
\end{tabular}

IQR: interquartile range. 


\section{DISCUSSION}

Infections are one of the most common complications of ventricular CSF shunts. ${ }^{1}$ The incidence of surgery-associated ventriculitis has been reported to range from $1 \%$ to $23 \%^{3}$ and to have reduced in recent years following the introduction of different strategies aimed at preventing surgical site infections. In the United States and Canada, rates have been reported to be below $5.7 \% .{ }^{9}$ However, other countries such as Turkey and Korea have reported rates of $15.8 \%{ }^{10}$ and $10.5 \%{ }^{11}$, respectively. In our hospital, the rates of infection were $4.37 \%$ in $2012,5.16 \%$ in $2013,3.41 \%$ in 2014 , and $6.5 \%$ in 2015 . The wide variability of rates reported in the bibliography is affected by the different diagnostic criteria used in each study. Some studies, including this one, ${ }^{11-13}$ only consider CSF culture positive results, whereas others include CSF cultures and / or cytochemistry alterations. ${ }^{5,9,10,14}$ In 2015, the Centers for Disease Control and Prevention $(\mathrm{CDC})^{15}$ changed the diagnostic criteria for ventriculitis and, at present, in addition to the microbiological criterion, compatible clinical manifestations, cytochemistry alterations, positive Gram staining or blood culture isolation are required, although the Infectious Diseases Society of America (IDSA) 2017 guidelines for health care-associated ventriculitis and meningitis states that CSF culture is the most important test for diagnosis. ${ }^{4}$

In relation to risk factors, most cases in this series occurred within 30 days of shunt implantation, which was consistent with previous reports, ${ }^{10}$ and supported the fact that the mechanism most likely involved was shunt colonization during the surgical procedure. ${ }^{4}$ Almost half of patients had a history of one or more valve replacements and almost one third had a prior shunt-associated infection. Such history has been associated with the risk for a new infection in several series. ${ }^{4,16,17}$ No association was observed between the current microorganism and the agent that caused the prior infection; only $3(5 \%)$ patients had recurrence, unlike other studies that have reported a higher recurrence incidence. ${ }^{3,10}$

Valve dysfunction, without signs of meningitis, was the most common presentation, in agreement with the bibliography. It is common to see only minimal ventriculitis without meningeal involvement or only mechanical dysfunction as a result of biofilm formation in or on the catheter. ${ }^{5}$ The studies by J. K. Lee et al. and Turgut et al. reported that $91 \%$ and $77 \%$ of patients had fever, respectively, ${ }^{11,18}$ whereas in our series fever was present in less than half of cases. Cellulitis along the tract was observed only in 6 infections $(11 \%)$, similar to what was reported by Turgut et al. $(11.4 \%)^{18}$

The CSF culture at the time of admission showed pathological findings in the case of $42(78 \%)$ infections, similar to what has been observed by Conen et al., who analyzed 78 infections and found that $80 \%$ had a leukocyte count $>5$ cells $/ \mathrm{mL}$ in CSF, whereas $20 \%$ had a normal cytochemistry. ${ }^{5}$

The presence of a normal cytochemistry in a patient suspected to have a ventricular CSF shunt-associated infection does not rule it out. CSF culture is the gold standard for ventriculitis diagnosis. In relation to microbiology, Grampositive microorganisms predominated, whereas a reduction in Gram-negative microorganisms was observed, and fungal infections were rare. This finding is consistent with what has been previously reported in our hospital and recently by other studies. ${ }^{9-11,16}$ However, other studies disagree with this and have reported a higher incidence of Gram-negative bacilli. ${ }^{19}$

Almost all infections were managed with surgical treatment, which included ventricular shunt removal plus systemic antibiotic therapy, consistent with the recommendations and prior studies that reported a greater level of success with this strategy. ${ }^{1,20}$

Mortality caused by ventricular CSF shuntassociated infections has been reported to be $10-13 \%{ }^{10,18,21}$ Other studies have described higher prevalence values, as those by Bathia et al. $(15 \%),{ }^{12}$ Kanik et al. $(15.8 \%),{ }^{10} \mathrm{Kim}$ et al. $(17 \%),{ }^{13}$ and Guardado et al. (33\%);22 however, others have reported lower rates, such as Ochieng $(4 \%){ }_{1}^{14}$ J. K. Lee et al. $(2.85 \%),{ }^{11}$ and Wong et al. $(6 \%) .{ }^{23}$

In our series, mortality was $9 \%$, similar to that described in the study by Srihawan et al. ${ }^{9}$ Such variability is mostly influenced by 2 factors: the type of patients predominating in each series (those with a higher percentage of patients with CNS tumors show higher rates), and series with a high prevalence of resistant Gram-negative bacilli. ${ }^{21,22}$

In the study by Gmeiner et al., ${ }^{2}$ who analyzed the causes of death in the long term in patients with ventricular CSF shunts, $78 \%$ of related deaths were caused by an infection and not by a shunt and short-circuit dysfunction. This was consistent with the study by Tuli et al., ${ }^{24}$ who 
assessed 907 pediatric patients (children with CNS tumors were excluded) and concluded that a short-circuit-associated infection was a predictor of death.

All deaths in this series occurred within 1 month of infection diagnosis; 2 patients died within the first 48 hours of hospitalization due to septic shock and the other 3 died due to progression of the underlying disease associated with the infection. Following a univariate analysis, the only statistically significant factors associated with mortality were positive blood cultures, fever at the time of admission, and septic shock. Few studies conducted a multivariate analysis of predictors of mortality: a study by Kim et al. ${ }^{13}$ included 91 adult patients with a mortality rate of $16.5 \%$ and associated factors were failure to remove the shunt and septic shock. The largest study included 215 children and adults with health care-associated meningitis and ventriculitis ${ }^{9}$ and its objective was to assess predictors of an unfavorable clinical course. The mortality rate was $9.3 \%$ and the logistic regression analysis identified that age older than 45 years, an abnormal neurological exam, and mechanical ventilation were independent risk factors for an adverse outcome.

One of the weaknesses of this study was its sample size, which may have affected its statistical power to establish group differences. Another limitation of this study was its retrospective nature. In turn, since the study was conducted in a tertiary care hospital, results may not be extrapolated to other sites. The strength of this study was that, to our knowledge, it is one of the few updated studies conducted in Argentina in the pediatric population with ventricular CSF shunts that analyzed infection characteristics and risk factors for mortality in relation to this device.

\section{CONCLUSIONS}

Valve dysfunction, fever, and cellulitis along the tract were the most common clinical manifestations of ventricular CSF shuntassociated infections.

Coagulase-negative Staphylococcus was the most common microorganism. Valve removal plus antibiotic therapy was the most frequently instituted treatment.

Fever at the time of admission, positive blood cultures, and septic shock were predictors of mortality.

\section{REFERENCES}

1. Flannery AM, Mitchell L. Pediatric hydrocephalus: systematic literature review and evidence-based guidelines. Part 1: Introduction and methodology. J Neurosurg Pediatr 2014;14(Suppl 1):3-7.

2. Gmeiner M, Wagner $H$, Zacher C, et al. Long-term mortality rates in pediatric hydrocephalus: a retrospective singlecenter study. Childs Nerv Syst 2017;33(1):101-9.

3. Ram Y, Tina QT. Infections Related to Prosthetic or Artificial Devices. In: Cherry J. Demmler-Harrison G, Kaplan S, et al. Feigin and Cherry's Textbook of Pediatric Infectious Diseases. $7^{\text {th }}$ ed. Philadelphia, PA: Elsevier-Saunders; 2014. Pages 1029-30.

4. Tunkel AR, Hasbun R, Bhimraj A, et al. 2017 Infectious Diseases Society of America's Clinical Practice Guidelines for Healthcare-Associated Ventriculitis and Meningitis. Clin Infect Dis 2017;64(6):e34-65.

5. Conen A, Walti L, Merlo A, et al. Characteristics and Treatment Outcome of Cerebrospinal Fluid ShuntAssociated Infections in Adults: A Retrospective Analysis over an 11-Year Period. Clin Infect Dis 2008;47(1):73-82.

6. Hasbun R. Central Nervous System Device Infections. Curr Infect Dis Rep 2016;18(11):34.

7. Clark R, Lewinski M, Loeffelholz M, et al. Cumitech 31A: Verification and Procedures in the Clinical Microbiology Laboratory. Washington, DC: ASM Press; 2009.

8. Forbes BA, Granato PA. Processing specimens for bacteria. In: Murray P, Baron M, Pfaller F, et al edits. Manual of clinical microbiology. $6^{\text {th }}$ ed. Washington, DC: ASM Press; 1995.P.101-3.

9. Srihawan C, Castelblanco R, Salazar L, et al. Clinical characteristics and predictors of adverse outcomes in adult and pediatric patients with healthcare-associated ventriculitis and meningitis. Open Forum Infect Dis 2016;3(2):ofw077.

10. KanikA, Sırı N, Kose E, et al. Clinical and Economic Results of Ventriculoperitoneal Shunt Infections in Children. Turk Neurosurg 2015;(1):58-62.

11. Lee JK, Seok JY, Lee JH, et al. Incidence and Risk Factors of Ventriculoperitoneal Shunt Infections in Children: A Study of 333 Consecutive Shunts in 6 Years. J Korean Med Sci 2012;27(12):1563-8.

12. Bhatia PL, Lilani S, Shirpurkar R, et al. Coagulasenegative staphylococci: Emerging pathogen in central nervous system shunt infection. Indian J Med Microbiol 2017;35(1):120-3.

13. Kim H, Kim S, Park G, et al. The causes and treatment outcomes of 91 patients with adult nosocomial meningitis. Korean J Intern Med 2012;27(2):171-9.

14. Ochieng N, Okechi H, Ferson $S$, et al. Bacteria causing ventriculoperitoneal shunt infections in a Kenyan population. J Neurosurg Pediatr 2015;15(2):150-5.

15. CDC/NHSN Surveillance Definitions for Specific Types of Infections. January 2017. [Accessed on: April 11th, 2017]. Available at: https://www.cdc.gov/nhsn/pdfs/ pscmanual/17pscnosinfdef_current.pdf.

16. Rowensztein H, Manfrin L, Paglia M, et al. Características del líquido cefalorraquídeo en niños con infecciones del sistema de derivación ventrículo-peritonea. Arch Argent Pediatr 2015;113(3):244-7.

17. Simon T, Butler J, Whitlock K, et al. Risk factors for first cerebrospinal fluid shunt infection: findings from a multicenter prospective cohort study. J Pediatr 2014;164(6):14628.e2.

18. Turgut M, Alabaz D, Erbey F, et al. Cerebrospinal Fluid Shunt Infections in Children. Pediatr Neurosurg 2005;41(3):131-6. 
19. Stenehjem E, Armstrong WS. Central nervous system device infections. Infect Dis Clin North Am 2012;26(1):89-110.

20. Rosanova MT, Paganini H. Infecciones asociadas a sistemas de derivación de líquido cefalorraquídeo. Med Infant 1997;4(1):26-30.

21. Sacar $S$, Turgut $H$, Toprak $S$, et al. A retrospective study of central nervous system shunt infections diagnosed in a university hospital during a 4-year period. BMC Infect Dis 2006;6:43.
22. Rodríguez GuardadoA, Blanco A, Asensi V, etal.Multidrugresistant Acinetobacter meningitis in neurosurgical patients with intraventricular catheters: assessment of different treatments. J Antimicrob Chemother 2008;61(4):908-13.

23. Wong HT, Pu JK, Wong WK, et al. Primary ventriculoperitoneal shunting outcomes: a multicenter clinical audit for shunt infection and its risk factors. Hong Kong Med J 2016;22(5):410-9.

24. Tuli S, Tuli J, Drake J, et al. Predictors of death in pediatric patients requiring cerebrospinal fluid shunts. J Neurosurg 2004;100(5 Suppl Pediatrics):442-6. 